\title{
A Novel Method for the Approximation of Humeral Head Retrotorsion Based on Three-Dimensional Registration of the Bicipital Groove
}

\author{
Vlachopoulos, Lazaros ; Carrillo, Fabio ; Dünner, Celestine ; Gerber, Christian ; Székely, Gábor ;
} Fürnstahl, Philipp

\begin{abstract}
BACKGROUND The accurate restoration of premorbid anatomy is key for the success of reconstructive surgeries of the proximal part of the humerus. The bicipital groove has been proposed as a landmark for the prediction of humeral head retrotorsion. We hypothesized that a novel method based on bilateral registration of the bicipital groove yields an accurate approximation of the premorbid anatomy of the proximal part of the humerus. METHODS Three-dimensional (3D) triangular surface models were created from computed tomographic data of 100 paired humeri (50 cadavers). Segments of the distal part of the humerus and the humeral shaft of prespecified lengths were defined. A surface registration algorithm was applied to superimpose the models onto the mirrored contralateral humeral model based on the defined segments. We evaluated the 3D proximal humeral contralateral registration (p-HCR) errors, defined as the difference in 3D rotation of the humeral head between the models when superimposed. For comparison, we quantified the landmark-based retrotorsion (LBR) error, defined as the intra-individual difference in retrotorsion, measured with a landmark-based 3D method. RESULTS The mean 3D p-HCR error using the most proximal humeral shaft (bicipital groove) segment for the registration was $2.8^{\circ}$ (standard deviation $[\mathrm{SD}], 1.5^{\circ}$; range, $0.6^{\circ}$ to $7.4^{\circ}$ ). The mean $\mathrm{LBR}$ error of the reference method was $6.4^{\circ}\left(\mathrm{SD}, 5.9^{\circ}\right.$; range, $0.5^{\circ}$ to $\left.24.0^{\circ}\right)$. CONCLUSIONS Bilateral 3D registration of the bicipital groove is a reliable method for approximating the premorbid anatomy of the proximal part of the humerus. CLINICAL RELEVANCE The accurate approximation of the premorbid anatomy is a key for the successful restoration of the premorbid anatomy of the proximal part of the humerus.
\end{abstract}

DOI: https://doi.org/10.2106/JBJS.17.01561

Posted at the Zurich Open Repository and Archive, University of Zurich

ZORA URL: https://doi.org/10.5167/uzh-153676

Journal Article

Published Version

Originally published at:

Vlachopoulos, Lazaros; Carrillo, Fabio; Dünner, Celestine; Gerber, Christian; Székely, Gábor; Fürnstahl, Philipp (2018). A Novel Method for the Approximation of Humeral Head Retrotorsion Based on ThreeDimensional Registration of the Bicipital Groove. Journal Bone Joint Surgery America, 100(15):e101.

DOI: https://doi.org/10.2106/JBJS.17.01561 


\title{
A Novel Method for the Approximation of Humeral Head Retrotorsion Based on Three-Dimensional Registration of the Bicipital Groove
}

\author{
Lazaros Vlachopoulos, MD, PhD, Fabio Carrillo, MSc, Celestine Dünner, MSc, Christian Gerber, MD, FRCSEd(Hon), \\ Gábor Székely, PhD, and Philipp Fürnstahl, PhD \\ Investigation performed at Balgrist University Hospital, Zurich, Switzerland
}

\begin{abstract}
Background: The accurate restoration of premorbid anatomy is key for the success of reconstructive surgeries of the proximal part of the humerus. The bicipital groove has been proposed as a landmark for the prediction of humeral head retrotorsion. We hypothesized that a novel method based on bilateral registration of the bicipital groove yields an accurate approximation of the premorbid anatomy of the proximal part of the humerus.

Methods: Three-dimensional (3D) triangular surface models were created from computed tomographic data of 100 paired humeri (50 cadavers). Segments of the distal part of the humerus and the humeral shaft of prespecified lengths were defined. A surface registration algorithm was applied to superimpose the models onto the mirrored contralateral humeral model based on the defined segments. We evaluated the 3D proximal humeral contralateral registration ( $p$-HCR) errors, defined as the difference in 3D rotation of the humeral head between the models when superimposed. For comparison, we quantified the landmark-based retrotorsion (LBR) error, defined as the intra-individual difference in retrotorsion, measured with a landmark-based 3D method.
\end{abstract}

Results: The mean 3D p-HCR error using the most proximal humeral shaft (bicipital groove) segment for the registration was $2.8^{\circ}$ (standard deviation [SD], $1.5^{\circ}$; range, $0.6^{\circ}$ to $7.4^{\circ}$ ). The mean LBR error of the reference method was $6.4^{\circ}$ (SD, $5.9^{\circ}$; range, $0.5^{\circ}$ to $24.0^{\circ}$ ).

Conclusions: Bilateral 3D registration of the bicipital groove is a reliable method for approximating the premorbid anatomy of the proximal part of the humerus.

Clinical Relevance: The accurate approximation of the premorbid anatomy is a key for the successful restoration of the premorbid anatomy of the proximal part of the humerus.

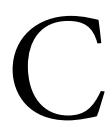
omputer-assisted methods have become increasingly important for the restoration of the premorbid anatomy of the proximal part of the humerus, as they support the surgeon in achieving this goal. Three-dimensional (3D) preoperative planning and intraoperative navigations techniques have been, thereby, proposed for proximal humeral fractures ${ }^{1-3}$ and corrective osteotomies of the proximal part of the humerus $^{4-6}$ as well as for shoulder replacement surgeries ${ }^{4,5,7-11}$.

In computer-assisted reconstructive surgery, the assessment of a deformity often relies on a comparison of the pathological bone with a $3 \mathrm{D}$ reconstruction template representing the normal anatomy. With respect to humeral deformities, the contralateral humeral anatomy has been accepted as a reliable reconstruction template ${ }^{6,12,13}$. A surface registration method is applied to superimpose the model of the pathological side onto the mirrored model of the contralateral side $e^{6,12}$. In the clinical setting, for example, the proximal part of the humerus might be pathological (i.e., fractured or malunited). The humeral segment distal to the pathological area can be used for the registration onto the contralateral side.

However, and especially if there are underlying side-toside differences in the twist about the humeral axis (humeral torsion) in a given individual ${ }^{14,15}$, these template-based approaches using the contralateral anatomy might introduce an error into the

Disclosure: This work was funded, in part, by the Swiss Canton of Zurich, Switzerland, through a Highly-Specialized Medicine grant and by the Balgrist Foundation, Zurich, Switzerland. One author (P.F.) is a shareholder of Balgrist CARD (Computer Assisted Research and Development), which develops preoperative planning software. The Disclosure of Potential Conflicts of Interest forms are provided with the online version of the article (http://links. Iww.com/JBJS/E849). 
The Journal of Bone \& Joint Surgery - Jbjs. org Volume 100-A · Number 15 -August 1, 2018
Approximation of Humeral Head Retrotorsion Based on 3D Registration of the Bicipital Groove quantification of the deformity. A recent study by our group demonstrated that, for the 3D assessment of distal humeral deformities, a proper segment selection of a healthy part of the humerus yields a deformity assessment of the distal part of the humerus that is less prone to bias arising from inherent bilateral differences ${ }^{16}$. Furthermore, several authors have proposed the bicipital groove to be a reliable intraoperative landmark for the restoration of the humeral retrotorsion during shoulder replacement surgery ${ }^{17-19}$.

We hypothesized that $3 \mathrm{D}$ registration of the proximal humeral shaft (inclusive of the bicipital groove) allows an accurate approximation of the premorbid proximal humeral anatomy. We systematically selected different segments of the distal part of the humerus and the humeral shaft for inclusion in the registration, and we evaluated the effect of these different segments on the robustness against bias due to underlying bilateral differences.

\section{Materials and Methods}

The Swiss Institute for Computer Assisted Surgery (SICAS) provided computed tomography (CT) full-body data for 50 cadavers (including the entire humerus of both sides). The in-plane (xy) resolution of the CT scans ranged from pixel sites of $0.9 \times 0.9$ to $1.27 \times 1.27 \mathrm{~mm}$. The slice thickness varied from 0.5 to $0.6 \mathrm{~mm}$. The average age (and standard deviation) of the individuals was $52.1 \pm 20.0$ years (range, 19 to 90 years). There were 32 male and 18 female donors. The average height was $172.4 \pm 8.7 \mathrm{~cm}$ (range, 154 to $187 \mathrm{~cm}$ ), and the average weight was $68.4 \pm 16.9 \mathrm{~kg}$ (range, 37 to $108 \mathrm{~kg}$ ). The specimens were also used in previous studies ${ }^{5,15,16}$. Exclusion criteria were the presence of osteoarthritis or previous trauma. Segmentation of the humerus was performed fully automatically using a previous described segmentation algorithm ${ }^{20}$. A marching cubes algorithm ${ }^{21}$ was applied to generate bilateral, 3D triangular surface models. Thereafter, the models were imported into the planning software CASPA (Computer Assisted Surgery Planning Application) that was developed in-house (Balgrist CARD [Computer Assisted Research and Development]).

\section{Current State-of-the-Art Deformity Assessment}

For the assessment of a posttraumatic deformity in 3 dimensions, the bone fragments of the pathological model (Fig. 1-A, source model) are reduced to a $3 \mathrm{D}$ reconstruction template (Fig. 1-A, target model, i.e., the mirrored contralateral model $)^{6,12,16,22,23}$. Two segments are selected on the source model, 1 proximal and 1 distal to the deformity (Fig. 1-B). A surface registration method, the iterative closest point (ICP) algorithm $^{24,25}$, is applied, to first superimpose the source model, based on the distal segment, onto the target model ${ }^{6,12,22,23}$. The target model corresponds to the reconstruction template after the distal registration. The deviation of the humeral head between the source model and the reconstruction template
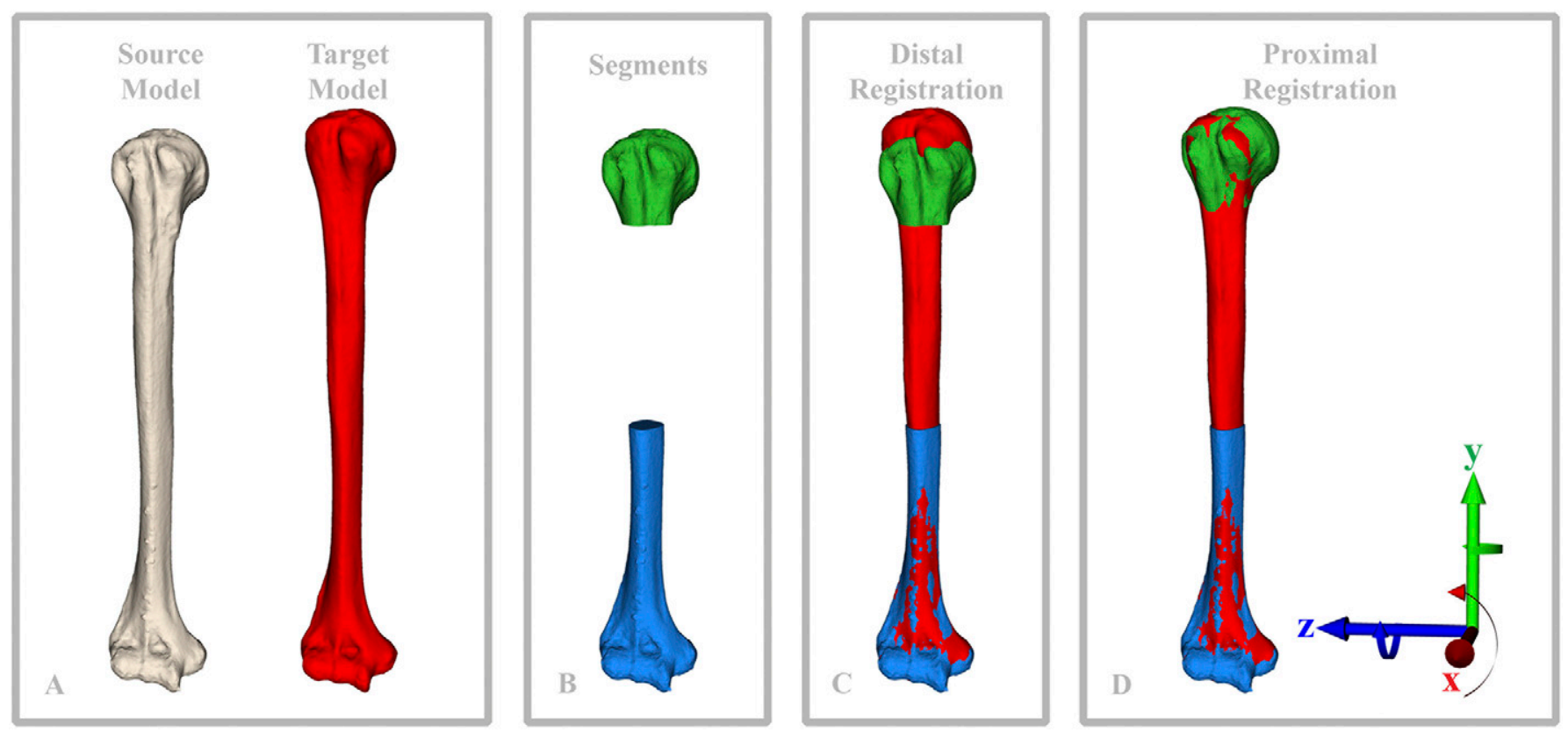

Fig. 1

Figs. 1-A through 1-D Assessment of posttraumatic deformity of the proximal part of the humerus. Fig. 1-A Three-dimensional bone models are generated, including the pathological humerus (source model) with the posttraumatic deformity after a proximal humeral fracture and the mirrored contralateral humeral model (target model), which serves as a reconstruction template. Fig. 1-B Two segments are selected distal (blue segment) and proximal (green segment) to the pathological area. Fig. 1-C After registration of the source model with the ICP algorithm based on the selected distal segment, the posttraumatic deformity corresponds to the deviation of the humeral head between the source (green segment) and the target model. Fig. 1-D To quantify the deformity, the ICP algorithm is applied to superimpose the humeral head segment of the source model onto the target model. 
The Journal of Bone \& Joint Surgery • Jbjs. org Volume 100-A · Number 15 -August 1, 2018
Approximation of Humeral Head Retrotorsion Based on 3D Registration of the Bicipital Groove corresponds to the deformity (Fig. 1-C). To measure the deformity, the ICP algorithm is applied to superimpose the proximal segment of the source model (humeral head segment) onto the reconstruction template. The relative $3 \mathrm{D}$ rotation and translation of the head segment between the distal (Fig. 1-C) and the proximal registration quantifies the malalignment (Fig. 1-D). We defined the 3D proximal humeral contralateral registration $(\mathrm{p}-\mathrm{HCR})$ error as the $3 \mathrm{D}$ rotational difference between the source and the target models of the proximal humeral anatomy after the distal registration.

\section{Effect of Segment Selection on the Approximation of Humeral Head Retrotorsion}

The principle of the ICP algorithm is to superimpose a source model onto a target model (Fig. 2) in such a way that the difference between the surfaces of the models is 0 (equal model surfaces) or minimal (varying model surfaces) within a userselected segment of interest ${ }^{16}$. We recently demonstrated that the selection of segments with idiosyncratic features as close as possible to the area of interest is relevant for registration-based approaches to compensate for bilateral differences ${ }^{16}$. In Figure 2, using a simplified representation, we illustrate the effect of segment selection on the assessment of humeral head retrotorsion when bilateral differences in humeral torsion are present without any pathological condition. The simplified humeral representation consists of 3 prisms, aligned axially along a cylinder. An elliptical cylinder (green) represents the humeral head, while cubes represent the distal part (blue) of the humerus and the proximal part of the shaft (yellow). The difference between the segments in retrotorsion is illustrated by the difference in rotation of the prisms around the cylinder (Fig. 2-A). The registration algorithm superimposes the models in a way that the surfaces of both models are the most similar for the particular selected segment (Figs. 2-B and 2-C). The influence of segment selection on the p-HCR error is illustrated in Figure 2-D.

In the present study, we used bilateral humeral models without a pathological condition, as this is the only way possible to analyze to effect of segment selection on the approximation of

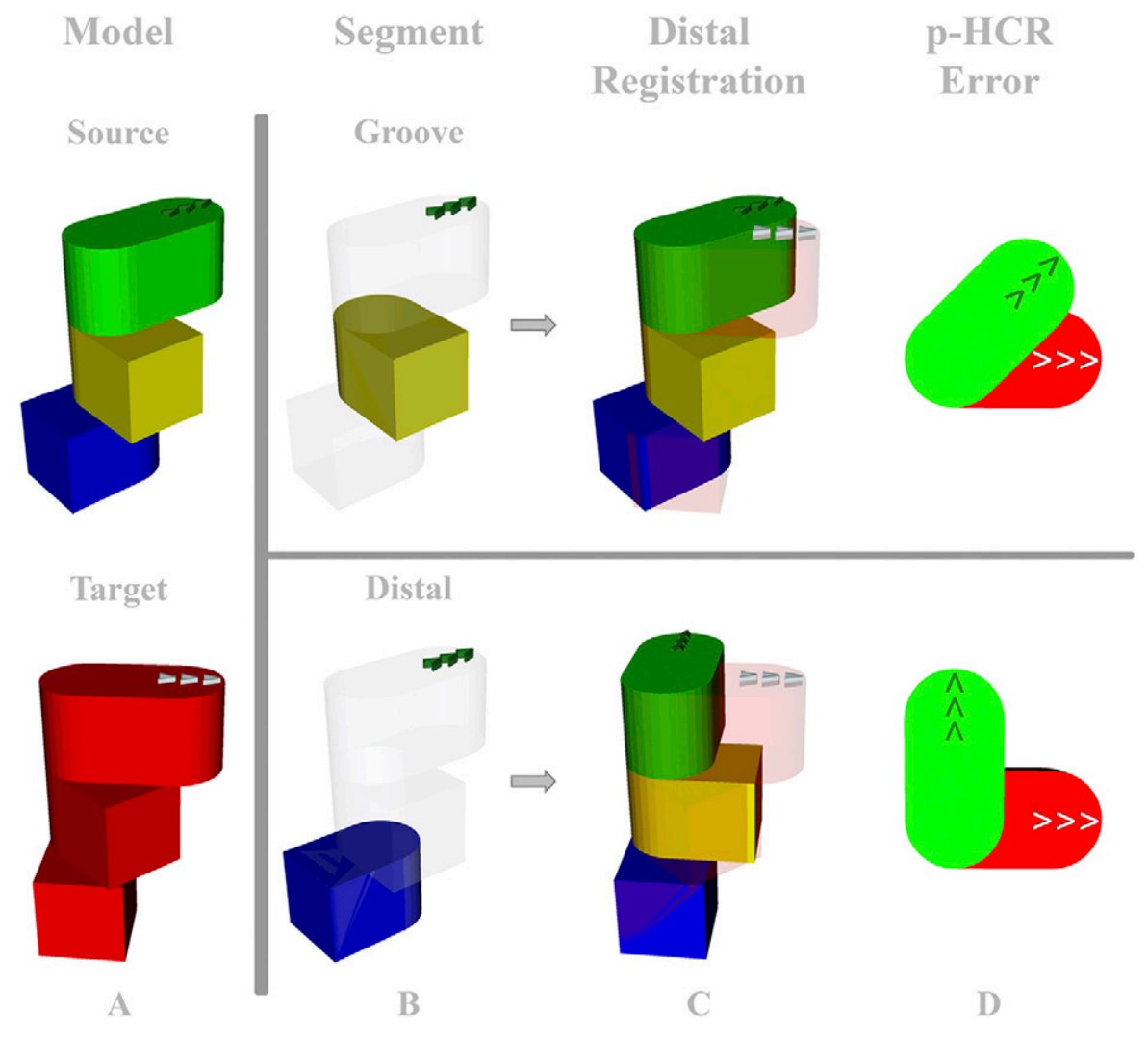

Fig. 2

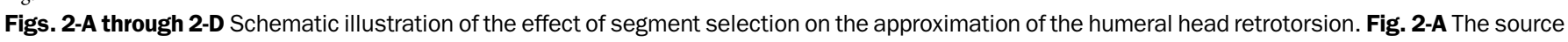

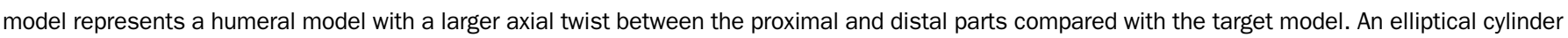

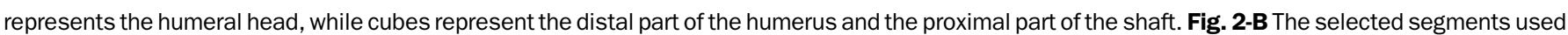

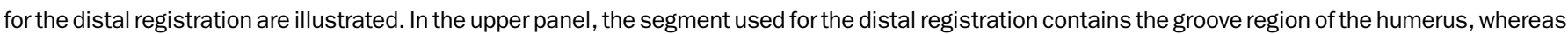

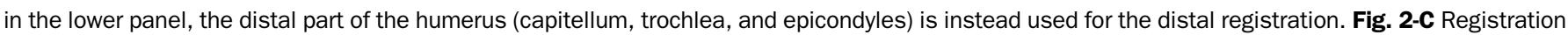

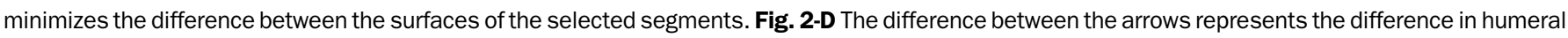

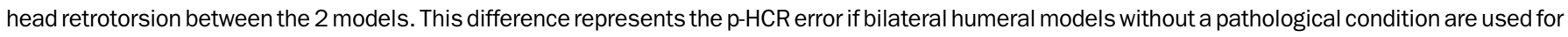
the assessment. 
The Journal of Bone \& Joint Surgery $\cdot$ JbJs.org Volume 100-A · Number $15 \cdot$ August 1, 2018
Approximation of Humeral Head Retrotorsion Based on 3D Registration of the Bicipital Groove the humeral head retrotorsion. In an ideal case, the deviation of the humeral head segments between both sides should be 0 . To determine the optimal segment, which allows superimposing the humeri in such a way that the (premorbid) humeral head retrotorsion is best approximated, we performed several registration experiments. We defined humeral segments on the basis of predetermined percentages of humeral length (Fig. 3-A). These segments were separated into 2 groups, according to anatomical region: the "groove group," which included 3 segments just below the humeral head, capturing the proximal part of the humeral shaft and the bicipital groove without the humeral head, and the "distal group," to which we assigned all segments including at least the distal part of the humerus (capitulum, trochlea, and epicondyles) (Figs. 3-B, 3-C, and 3-D).
We defined the right humerus as the source model. The left, contralateral humerus was mirrored about the sagittal plane of the coordinate system and served as the target model. To demonstrate that the evaluation was not biased by the selection of the source and target models, we repeated all computational experiments using the left humerus as the source model and the right humerus as the target model. This allowed assessment of the consistency of the method using the interclass correlation coefficient (ICC).

We divided each source model for the registration into the following 8 segments: groove-75-85, groove-50-85, and groove-50-75 (groove group), and dist-15, dist-25, dist-50, dist-75, and dist-85 (distal group) (Figs. 3-C and 3-D).

These segments were used to perform distal registrations as described above and shown in Figure 2-C. Hence, we

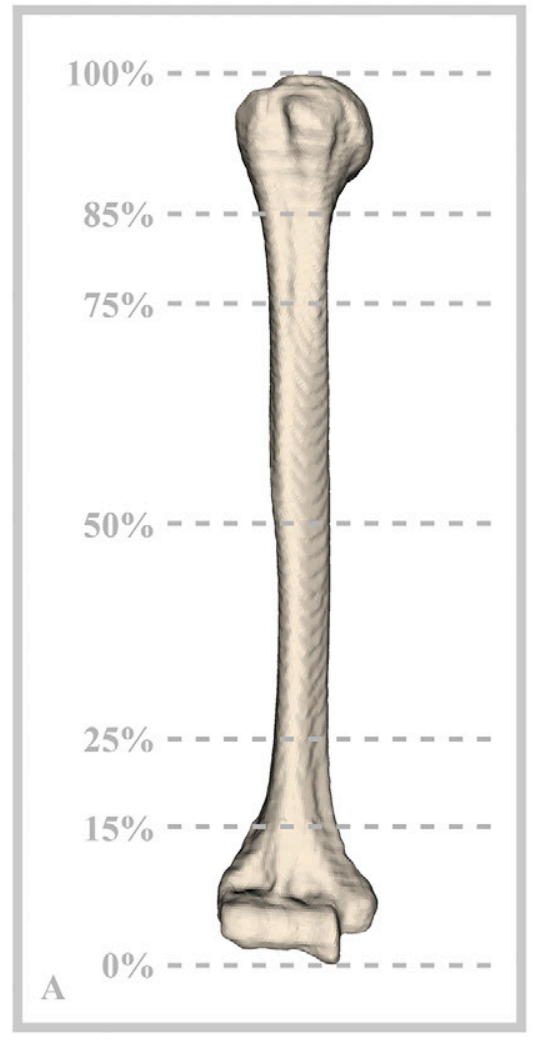

D
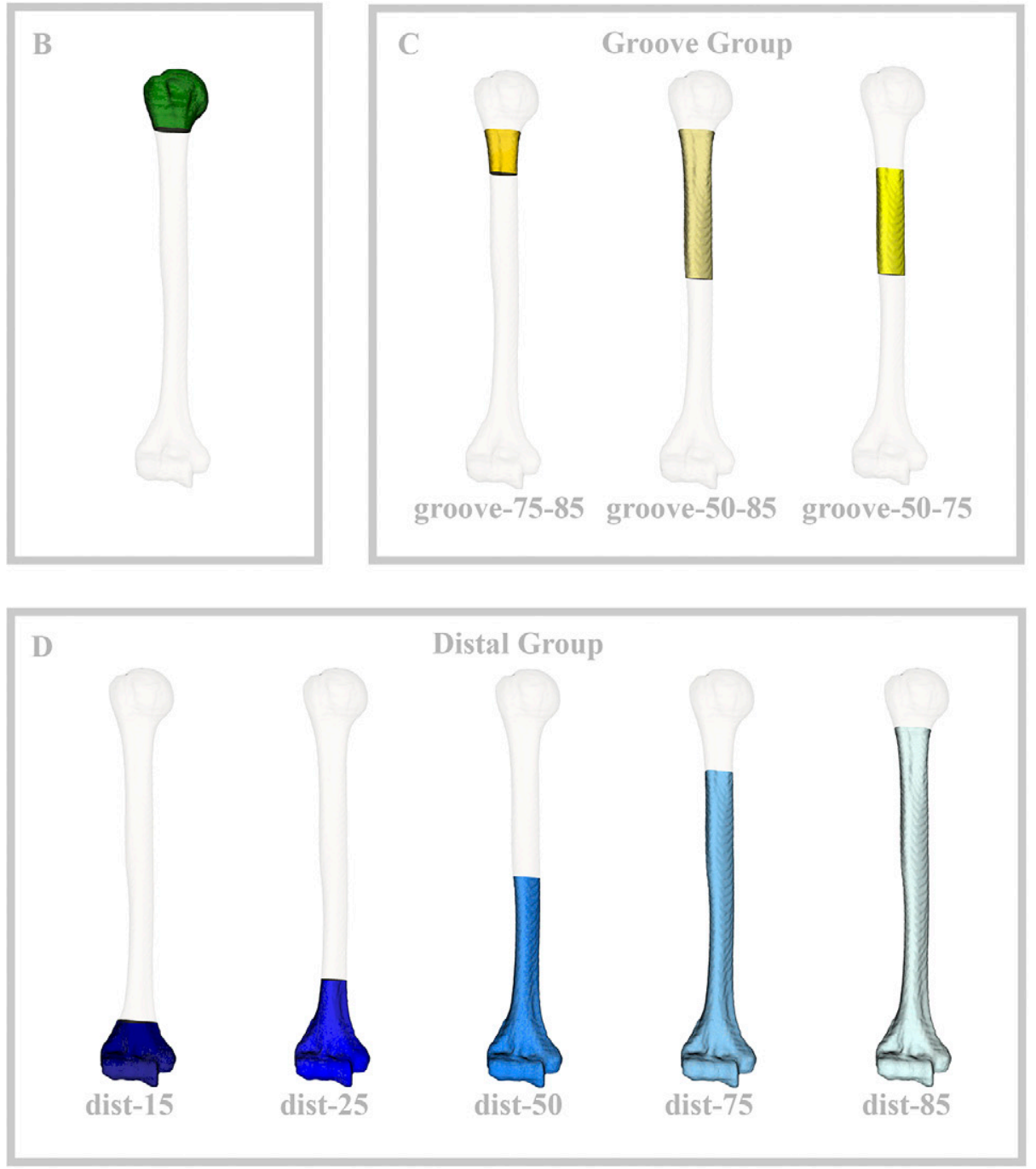

Fig. 3

Figs. 3-A through 3-D Generation of the segments defined for the registration. Fig. 3-A Right humeral model, with the humeral length expressed as a percentage from the distal end (0\%) to the proximal end (100\%). Fig. 3-B The defined segment for the evaluation of the p-HCR error (humeral head segment, in green). Figs. 3-C and 3-D The segments used for the distal registration: the groove group (Fig. 3-C) and the distal group (Fig. 3-D). Note that throughout the article, we use the same color code to represent the defined segments. 

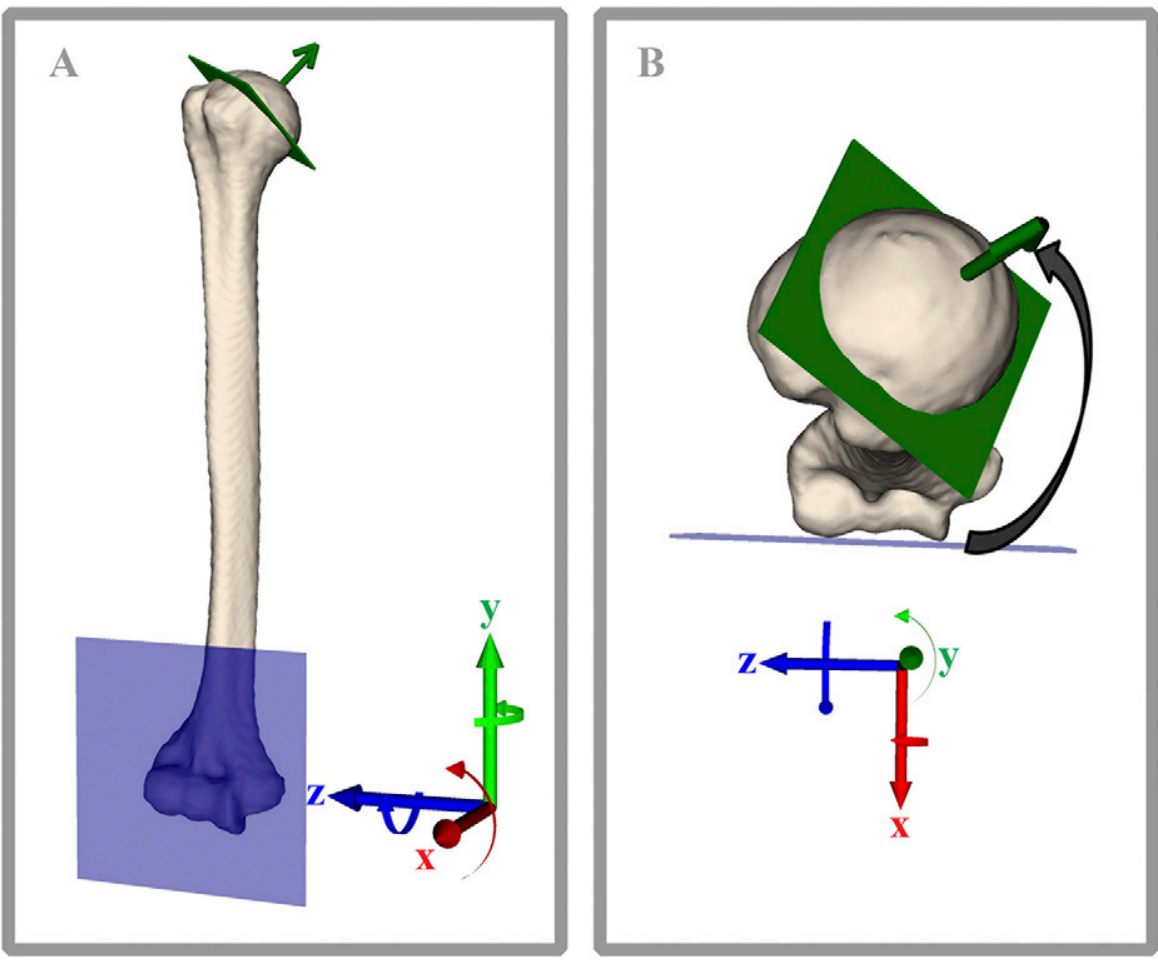

Fig. 4

Figs. 4-A and 4-B Landmark-based retrotorsion (LBR) error. Anteroposterior view (Fig. 4-A) and axial view (Fig. 4-B) of a humeral model with the elbow tangent plane (blue plane), the articular margin plane (green plane with arrow indicating the normal vector of the plane), and the humeral coordinate system. For the calculation of the LBR, the normal vector of the elbow tangent plane and the normal vector of the articular margin plane are projected onto the $x-z$ plane of the humeral coordinate system. The angle between those 2 projected vectors minus $90^{\circ}$ yields the LBR angle as defined clinically. The LBR error is the absolute difference in the LBR angles of both sides of an individual.

obtained 8 different results for the superimposed humeral pairs. For each humeral pair, we performed 8 left-to-right and 8 rightto-left registrations, which yielded 800 registration experiments in total.

To quantify the 3D p-HCR errors, we consistently used a humeral head segment that, in all instances, involved $15 \%$ of the total length of the humerus, including the humeral head and the greater tuberosity (Fig. 3-B). A 3D p-HCR error of $0^{\circ}$ indicates that the premorbid retrotorsion of a pathological humerus could be approximated perfectly with the registrationbased approach using the selected segment. The p-HCR error is expressed in axis-angle representation (3D p-HCR error) and, additionally, as 3 constitutive rotations (i.e., Euler angles). All measurements were performed with respect to the standardized

TABLE I Values of the 3D p-HCR Error for the Proximal Part of the Humerus by Selected Segment and the Corresponding ICC*

\begin{tabular}{|c|c|c|c|c|c|c|c|c|c|c|c|c|}
\hline & \multicolumn{4}{|c|}{ Error $\left({ }^{\circ}\right)$} & \multirow[b]{2}{*}{ ICC (95\% Cl) } & \multicolumn{7}{|c|}{ P Value $†$} \\
\hline & Mean & Std. Dev. & Median & Range & & Groove-50-85 & Groove-50-75 & Dist-85 & Dist-75 & Dist-50 & Dist-25 & Dist-15 \\
\hline Groove-75-85 & 2.8 & 1.5 & 2.4 & $0.6-7.4$ & $0.96(0.93-0.98)$ & 0.045 & 0.001 & $<0.0001$ & $<0.0001$ & $<0.0001$ & $<0.0001$ & $<0.0001$ \\
\hline Groove-50-75 & 4.4 & 2.8 & 3.3 & $0.9-12.8$ & $0.98(0.96-0.99)$ & & & $<0.001$ & 0.001 & $<0.001$ & $<0.001$ & $<0.001$ \\
\hline Dist-85 & 6.5 & 4.7 & 5.5 & $0.8-23.5$ & $1.00(0.99-1.00)$ & & & & 0.10 & $<0.0001$ & $<0.0001$ & $<0.0001$ \\
\hline Dist-75 & 6.5 & 4.6 & 5.5 & $0.8-20.4$ & $0.99(0.98-0.99)$ & & & & & $<0.0001$ & $<0.0001$ & $<0.0001$ \\
\hline Dist-25 & 7.1 & 4.5 & 6.3 & 1.3-18.7 & $1.00(0.99-1.00)$ & & & & & & & $<0.001$ \\
\hline Dist-15 & 7.4 & 4.5 & 6.2 & $1.6-19.3$ & $1.00(0.99-1.00)$ & & & & & & & \\
\hline
\end{tabular}

*Friedman rank-sum test revealed a significant effect of the selected segments on the $3 \mathrm{D} p$-HCR Error $(142.9 ; \mathrm{p}<0.0001)$. Cl $=$ confidence interval. $\dagger \mathrm{P}$ values from post-hoc analysis, conducted with Wilcoxon signed-rank test with Bonferroni correction (adjusted $p=0.05 / 28 \approx 0.0018$ ). 
TABLE II Values of the p-HCR Error Around the Y Axis (Internal/External Rotation) and the LBR Error*

\begin{tabular}{|c|c|c|c|c|c|c|c|c|c|c|c|c|}
\hline & \multicolumn{4}{|c|}{ Error $\left(^{\circ}\right)$} & \multicolumn{8}{|c|}{ P Value $\dagger$} \\
\hline & Mean & Std. Dev. & Median & Range & Groove-50-85 & Groove-50-75 & Dist-85 & Dist-75 & Dist-50 & Dist-25 & Dist-15 & LBR \\
\hline Groove-75-85 & 2.1 & 1.6 & 1.7 & $0.1-6.9$ & 0.045 & 0.001 & $<0.0001$ & $<0.0001$ & $<0.0001$ & $<0.0001$ & $<0.0001$ & $<0.0001$ \\
\hline Groove-50-75 & 3.6 & 2.9 & 2.5 & $0.4-11.2$ & & & $<0.001$ & 0.001 & $<0.001$ & $<0.001$ & $<0.001$ & 0.005 \\
\hline Dist-85 & 5.8 & 4.9 & 5.2 & $0.4-22.9$ & & & & 0.10 & $<0.0001$ & 0.0001 & $<0.0001$ & 0.45 \\
\hline Dist-75 & 5.8 & 4.8 & 4.9 & $0.4-19.8$ & & & & & $<0.0001$ & $<0.0001$ & $<0.0001$ & 0.40 \\
\hline Dist-15 & 6.4 & 4.8 & 5.7 & $0.2-18.3$ & & & & & & & & 0.98 \\
\hline LBR & 6.4 & 5.9 & 5.2 & $0.5-24.0$ & & & & & & & & \\
\hline
\end{tabular}

*Friedman rank-sum test revealed a significant effect of the selected segments on the $\mathrm{p}-\mathrm{HCR}$ Error $(95.4 ; \mathrm{p}<0.0001)$. $† \mathrm{P}$ values from post-hoc analysis, conducted with Wilcoxon signed-rank test with Bonferroni correction (adjusted $p=0.05 / 36 \approx 0.0014$ ).

coordinate system of the International Society of Biomechanics $(\mathrm{ISB})^{26}$. The rotation around the $\mathrm{y}$ axis (Fig. 1, green axis) corresponds, thereby, to a rotation in the axial plane (internal/external rotation).

\section{Landmark-Based Method (LBR Error)}

For comparison of the p-HCR errors based on the bilateral differences in humeral torsion, we assessed the landmark-based retrotorsion (LBR) error. We measured the retrotorsion of each humerus between the articular margin plane and the elbow tangent plane, as previously described (Fig. 4$)^{15}$. Consequently, the LBR error is the absolute difference in the retrotorsion angles of both sides of an individual.

The portion of the 3D p-HCR error involving rotation around the $y$ axis can be interpreted as internal/external rotation and is comparable with the LBR error. The portions of the $3 \mathrm{D}$-HCR error involving rotation around the $\mathrm{x}$ and $\mathrm{z}$ axes are not captured by the LBR error.

\section{Statistical Analysis}

A Mauchly sphericity test revealed a violation of the assumption of sphericity. Therefore, we applied the nonparametric Friedman rank-sum test, with the segment as a group factor and the individuals as a block factor, to analyze the effect of the selected segments on the (3D) p-HCR error. Post-hoc analysis was performed with the Wilcoxon signed-rank test with Bonferroni adjustment. Significance was set at the level of $\mathrm{p}<0.05$. The consistency of the method was assessed with the ICC (2way random-effects model). All reported p-HCR errors were calculated from the average of the absolute values, when the right or left humerus was selected as a source model.

\section{Results}

\section{$3 D$ p-HCR Error}

7 he 3D p-HCR error when using the groove-75-85 or 1 groove-50-85 segment for the registration was significantly smaller than when using any other segment. The difference between the groove-75-85 and groove-50-85 segments was not significant. The ICC was $\geq 0.96$ for all selected segments (Table I).

\section{P-HCR Error Around the Y Axis (Internal/External Rotation) and the LBR Error}

The p-HCR error around the y axis (internal/external rotation) when using the groove-75-85 or groove-50-85 segment for the registration was significantly smaller than when using the groove-50-75 segment or any segment of the distal group and significantly smaller than the LBR error. The p-HCR error around the $y$ axis (internal/external rotation) using the groove50-75 segment or any segment of the distal group did not differ significantly from the LBR error (Table II).

The percentage of individuals by category of error size $\left(<5^{\circ}, 5^{\circ}\right.$ to $10^{\circ}$, or $\left.>10^{\circ}\right)$ for the $\mathrm{p}$-HCR error around the $\mathrm{y}$ axis (internal/external rotation) and LBR error is shown in Table III.

\begin{tabular}{|l}
$\begin{array}{c}\text { TABLE III Rates by Category of Error Size for the p-HCR Error } \\
\text { Around the Y Axis (Internal/External Rotation) and } \\
\text { LBR Error* }\end{array}$ \\
\cline { 2 - 4 } \\
\cline { 2 - 4 }
\end{tabular}


The Journal of Bone \& Joint Surgery • Jbjs. org Volume 100-A · Number 15 -August 1, 2018
Approximation of Humeral Head Retrotorsion Based on 3D Registration of the Bicipital Groove

\section{Discussion}

$\mathrm{T}$ he template-based approach is the most commonly used method for the 3D assessment of posttraumatic deformities $^{6,12,13}$. Since differences exist in humeral head retrotorsion between both sides of an individual ${ }^{14,15}$, these template-based approaches, using the contralateral anatomy as a template, might introduce an error into the quantification of the deformity and may, thereby, compromise the ultimate goal of restoring the premorbid anatomy of the proximal part of the humerus.

Several authors proposed the bicipital groove as a reliable intraoperative landmark for the restoration of humeral retrotorsion during shoulder replacement surgery ${ }^{17-19}$. Furthermore, in a recent study, Johnson et al. ${ }^{27}$ analyzed the 3D shape of the bicipital groove and reported a significant correlation between bicipital groove rotation and humeral head retrotorsion. The authors presented equations for predicting humeral retrotorsion once the groove rotation was assessed. However, they pointed out that advanced methods, such as with computer-navigation technology, would be required for measuring the groove. Moreover, in their study, they did not perform a comparison between a gold standard and the approximation error resulting from the calculation of torsion using the equations presented.

In the present study, we demonstrated that bilateral 3D registration of the bicipital groove is an appropriate method for 3D approximation of the proximal humeral anatomy. The bicipital groove contains idiosyncratic features that allow the superimposing of the humeral models of both sides of an individual in such a way that the error in approximation of humeral head retrotorsion is smaller than the overall bilateral difference in retrotorsion for an individual. Figure 5 illustrates the crosssectional shape of the groove-75-85 segment.

The current study confirms our previous findings ${ }^{16}$ that the presence of idiosyncratic features is relevant for registration-
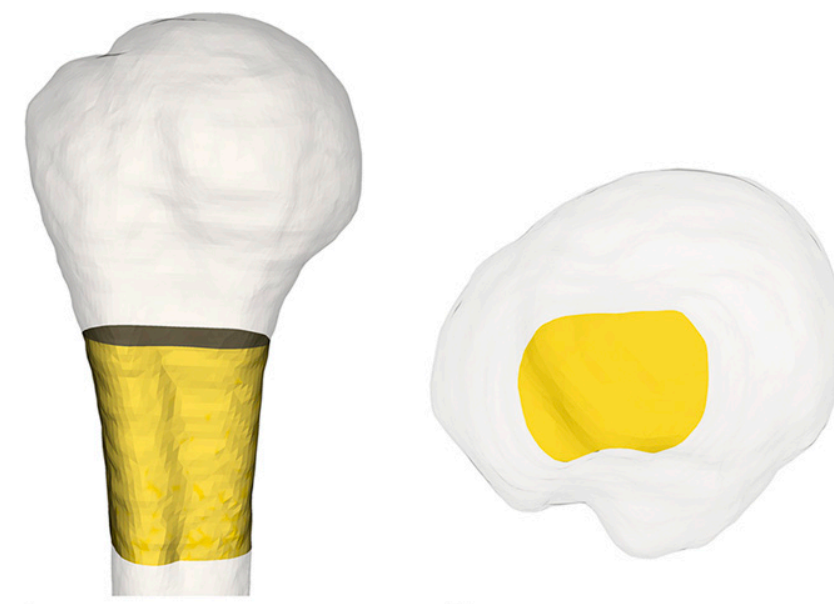

Fig. 5

Figs. 5-A and 5-B Groove-75-85 segment. The 3D shape (Fig. 5-A) of this segment and its cross-sectional shape (Fig. 5-B) indicate that the bicipital groove has a characteristic shape (i.e., in contrast to a cylindrical shape) that allows the superimposing of the humeral models with the ICP algorithm in a consistent way. based approaches that compensate for bilateral differences. However, if we used segments with the same distance to the assumed pathological area, the errors in approximating the proximal humeral anatomy were slightly higher in the current study than the errors in approximating the distal humeral anatomy in the previous study ${ }^{16}$. This confirms the previous conclusion that the magnitude of error and the consistency of registration have to be validated separately when considering implementing this new approach for other skeletal sites.

Recent studies by Poltaretskyi et al. ${ }^{4}$ and our group ${ }^{5}$ are closely related to the present work. In those studies, another approach was presented for the generation of a $3 \mathrm{D}$ reconstruction template: use of a statistical shape model (SSM). The results of the SSM with the "missing epiphysis and metaphysis"4 or of the dist- 85 segment ${ }^{5}$ can be compared with the results of the approximation of the proximal humeral anatomy with the groove-75-85 segment in the current study. The approximation of the proximal humeral anatomy with the bilateral registration of the bicipital groove seems to be more accurate than the SSM. The mean p-HCR error around the $y$ axis using the groove-7585 segment was $2.1^{\circ} \pm 1.6^{\circ}$, while the corresponding error in retrotorsion with the SSM of Poltaretskyi et al..$^{4}$ was $3.8^{\circ} \pm 2.9^{\circ}$. The $3 \mathrm{D}$ p-HCR in the present study using the groove- $75-85$ segment was $2.8^{\circ} \pm 1.5^{\circ}$, while the error of the SSM with the distal-85 segment ${ }^{5}$ was $3.8^{\circ} \pm 1.9^{\circ}$. Although these differences might not be clinically relevant, both methods have their benefits and limitations, as previously described ${ }^{5}$, and therefore, we believe that the 2 approaches are not mutually exclusive or competitive but rather, complementary.

As a reference, we also assessed the LBR error. The mean LBR error was $6.4^{\circ}$, which corresponds to the intra-individual bilateral differences in humeral head retrotorsion ${ }^{14,15}$. While the LBR error was $<5^{\circ}$ in almost half of the cases, in $26 \%$ of the individuals, the LBR error was between $5^{\circ}$ and $10^{\circ}$, and in an additional $26 \%$ of the cases, it was $>10^{\circ}$. The p-HCR errors around the $y$ axis (internal/external rotation) of the registrationbased approach using the segments of the distal group were similar to the LBR error. This indicates that, with registration of the distal segments, the humeral models are superimposed in a way that preserves the bilateral differences in retrotorsion. In contrast, the p-HCR errors of the groove group were significantly smaller than the p-HCR errors of the distal group and the LBR error. The difference between the groove- $50-85$ and groove- $75-85$ segments was not significant. If one of these groove segments was used for the distal registration, the p-HCR error was $>10^{\circ}$ in $\leq 2 \%$ of the cases. It should be pointed out that all of the reported errors in the current study represent only the deviation of the approximated anatomy (i.e., planned correction) relative to the premorbid anatomy. In a postoperative "worst-case scenario," the overall error in restoring the pretraumatic anatomy might increase with an additional error introduced during surgery.

One limitation of the present study was that preoperative assessment of a deformity can only be applied if the contralateral bone is healthy. However, the distal part of the humerus is not necessary for the distal registration. Therefore, the method can be used even in the presence of a pathological condition of the 
The Journal of Bone \& Joint Surgery • Jbjs.org Volume 100-A · Number $15 \cdot$ August 1, 2018
Approximation of Humeral Head Retrotorsion Based on 3D Registration of the Bicipital Groove distal humeral anatomy on the contralateral side. Furthermore, there still might be small differences in humeral head retrotorsion between the humeral head segments even if osseous landmarks of the humeral head segments of both sides were to be perfectly reconstructed. Nevertheless, in our opinion, it is preferable to use the proposed registration-based approach with the groove-75-85 or groove-50-85 segment for the 3D approximation of the proximal humeral anatomy.

In conclusion, bilateral $3 \mathrm{D}$ registration of the bicipital groove is a reliable method for approximating premorbid proximal humeral anatomy.

Lazaros Vlachopoulos, MD, $\mathrm{PhD}^{1,2}$

Fabio Carrillo, MSc ${ }^{1}$
Celestine Dünner, $\mathrm{MSc}^{2}$

Christian Gerber, MD, FRCSEd(Hon $)^{1}$

Gábor Székely, $\mathrm{PhD}^{2}$

Philipp Fürnstahl, $\mathrm{PhD}^{1}$

${ }^{1}$ Computer Assisted Research and Development Group (L.V., F.C., and P.F.) and Department of Orthopaedics (C.G.), Balgrist University Hospital, University of Zurich, Zurich, Switzerland

${ }^{2}$ Computer Vision Laboratory, ETH Zurich, Zurich, Switzerland

E-mail address for L. Vlachopoulos: lazaros.vlachopoulos@balgrist.ch

ORCID iD for L. Vlachopoulos: 0000-0001-8526-6032

ORCID iD for F. Carrillo: 0000-0002-0942-0277

ORCID iD for C. Dünner: $0000-0002-9880-7173$

ORCID iD for C. Gerber: 0000-0002-4624-8285

ORCID iD for G. Székely: $0000-0002-6560-8530$

ORCID iD for P. Fürnstahl: 0000-0001-6484-6206

\section{References}

1. Bicknell RT, DeLude JA, Kedgley AE, Ferreira LM, Dunning CE, King GJ, Faber KJ, Johnson JA, Drosdowech DS. Early experience with computer-assisted shoulder hemiarthroplasty for fractures of the proximal humerus: development of a novel technique and an in vitro comparison with traditional methods. J Shoulder Elbow Surg. 2007 May-Jun;16(3)(Suppl):S117-25. Epub 2007 Jan 17.

2. Fürnstahl P, Székely G, Gerber C, Hodler J, Snedeker JG, Harders M. Computer assisted reconstruction of complex proximal humerus fractures for preoperative planning. Med Image Anal. 2012 Apr;16(3):704-20. Epub 2010 Sep 29.

3. Vlachopoulos L, Székely G, Gerber C, Fürnstahl P. A scale-space curvature matching algorithm for the reconstruction of complex proximal humeral fractures. Med Image Anal. 2018 Jan;43:142-56. Epub 2017 Oct 27.

4. Poltaretskyi S, Chaoui J, Mayya M, Hamitouche C, Bercik MJ, Boileau P, Walch G. Prediction of the pre-morbid $3 \mathrm{D}$ anatomy of the proximal humerus based on statistical shape modelling. Bone Joint J. 2017 Jul;99-B(7):927-33.

5. Vlachopoulos L, Lüthi M, Carrillo F, Gerber C, Székely G, Fürnstahl P. Restoration of the patient-specific anatomy of the proximal and distal humerus: statistical shape modeling versus contralateral registration method. J Bone Joint Surg Am. 2018 Apr 18;100(8):e50.

6. Vlachopoulos L, Schweizer A, Meyer DC, Gerber C, Fürnstahl P. Three-dimensional corrective osteotomies of complex malunited humeral fractures using patient-specific guides. J Shoulder Elbow Surg. 2016 Dec;25(12):2040-7. Epub 2016 Aug 5.

7. Edwards TB, Gartsman GM, O'Connor DP, Sarin VK. Safety and utility of computer-aided shoulder arthroplasty. J Shoulder Elbow Surg. 2008 May-Jun;17(3): 503-8. Epub 2008 Feb 11.

8. Gauci MO, Boileau P, Baba M, Chaoui J, Walch G. Patient-specific glenoid guides provide accuracy and reproducibility in total shoulder arthroplasty. Bone Joint $\mathrm{J}$. 2016 Aug;98-B(8):1080-5.

9. Iannotti J, Baker J, Rodriguez E, Brems J, Ricchetti E, Mesiha M, Bryan J. Threedimensional preoperative planning software and a novel information transfer technology improve glenoid component positioning. J Bone Joint Surg Am. 2014 May 7; 96(9): 71

10. Kircher J, Wiedemann M, Magosch $P$, Lichtenberg $S$, Habermeyer P. Improved accuracy of glenoid positioning in total shoulder arthroplasty with intraoperative navigation: a prospective-randomized clinical study. J Shoulder Elbow Surg. 2009 Jul-Aug;18(4):515-20.

11. Tschannen M, Vlachopoulos L, Gerber C, Székely G, Fürnstahl P. Regression forest-based automatic estimation of the articular margin plane for shoulder prosthesis planning. Med Image Anal. $2016 \mathrm{Jul} ; 31: 88-97$. Epub 2016 Mar 3.

12. Murase $\mathrm{T}$, Oka K, Moritomo H, Goto A, Yoshikawa H, Sugamoto K. Three-dimensional corrective osteotomy of malunited fractures of the upper extremity with use of a computer simulation system. J Bone Joint Surg Am. 2008 Nov;90(11):2375-89.

13. Omori S, Murase T, Oka K, Kawanishi $\mathrm{Y}$, Oura K, Tanaka H, Yoshikawa $\mathrm{H}$. Postoperative accuracy analysis of three-dimensional corrective osteotomy for cubitus varus deformity with a custom-made surgical guide based on computer simulation. J Shoulder Elbow Surg. 2015 Feb;24(2):242-9. Epub 2014 Oct 25.
14. DeLude JA, Bicknell RT, Mackenzie GA, Ferreira LM, Dunning CE, King GJ, Johnson JA, Drosdowech DS. An anthropometric study of the bilateral anatomy of the humerus. J Shoulder Elbow Surg. 2007 Jul-Aug;16(4):477-83. Epub 2007 Mar 23. 15. Vlachopoulos L, Dünner C, Gass T, Graf M, Goksel O, Gerber C, Székely G, Fürnstahl P. Computer algorithms for three-dimensional measurement of humeral anatomy: analysis of 140 paired humeri. J Shoulder Elbow Surg. 2016 Feb;25(2): e38-48. Epub 2015 Oct 4.

16. Vlachopoulos L, Carrillo F, Gerber C, Székely G, Fürnstahl P. A novel registrationbased approach for 3D assessment of posttraumatic distal humeral deformities. J Bone Joint Surg Am. 2017 Dec 6;99(23):e127.

17. Doyle AJ, Burks RT. Comparison of humeral head retroversion with the humeral axis/biceps groove relationship: a study in live subjects and cadavers. J Shoulder Elbow Surg. 1998 Sep-Oct;7(5):453-7.

18. Hempfing A, Leunig M, Ballmer FT, Hertel R. Surgical landmarks to determine humeral head retrotorsion for hemiarthroplasty in fractures. J Shoulder Elbow Surg. 2001 Sep-Oct;10(5):460-3.

19. Kontakis GM, Damilakis J, Christoforakis J, Papadakis A, Katonis P, Prassopoulos $P$. The bicipital groove as a landmark for orientation of the humeral prosthesis in cases of fracture. J Shoulder Elbow Surg. 2001 Mar-Apr;10(2):136-9.

20. Gass T, Székely G, Goksel O. Simultaneous segmentation and multiresolution nonrigid atlas registration. IEEE Trans Image Process. 2014 Jul;23(7):2931-43. Epub 2014 May 7.

21. Lorensen WE, Cline HE. Marching cubes: a high resolution 3D surface construction algorithm. SIGGRAPH Comput Graph. 1987;21(4):163-9.

22. Kataoka T, Oka K, Miyake J, Omori S, Tanaka H, Murase T. 3-Dimensional prebent plate fixation in corrective osteotomy of malunited upper extremity fractures using a real-sized plastic bone model prepared by preoperative computer simulation. J Hand Surg Am. 2013 May;38(5):909-19. Epub 2013 Apr 6.

23. Schweizer A, Fürnstahl P, Nagy L. Three-dimensional correction of distal radius intra-articular malunions using patient-specific drill guides. J Hand Surg Am. 2013 Dec;38(12):2339-47. Epub 2013 Nov 1.

24. BesI PJ, McKay ND. A method for registration of 3-D shapes. IEEE Trans Pattern Anal Mach Intell. 1992;14(2):239-56.

25. Chen $\mathrm{Y}$, Medioni G. Object modeling by registration of multiple range images. Read at the 1991 IEEE International Conference on Robotics and Automation; 1991 Apr; Sacramento, CA.

26. Wu G, van der Helm FCT, Veeger HEJ, Makhsous M, Van Roy P, Anglin C, Nagels J, Karduna AR, McQuade K, Wang X, Werner FW, Buchholz B; International Society of Biomechanics. ISB recommendation on definitions of joint coordinate systems of various joints for the reporting of human joint motion-part II: shoulder, elbow, wrist and hand. J Biomech. 2005 May;38(5):981-92.

27. Johnson JW, Thostenson JD, Suva LJ, Hasan SA. Relationship of bicipital groove rotation with humeral head retroversion: a three-dimensional computed tomographic analysis. J Bone Joint Surg Am. 2013 Apr 17;95(8):719-24. 